\title{
Nano-particles Deposition in the Presence of Electric Field
}

Pouyan Talebizadeh Sardari ${ }^{1,2, *}$, Hassan Rahimzadeh $^{1}$, Goodarz Ahmadi ${ }^{3}$, Donald Giddings ${ }^{2}$

${ }^{1}$ Department of Mechanical Engineering, Amirkabir University of Technology, Iran.

${ }^{2}$ Fluids and Thermal Engineering Research Group, Faculty of Engineering, University of Nottingham, UK.

${ }^{3}$ Department of Mechanical and Aeronautical Engineering, Clarkson University, USA.

\begin{abstract}
The dispersion and deposition of nano-particles in laminar flows in the presence of an electric field were studied. The Eulerian-Lagrangian particle tracking method was used to simulate nano-particle motions under the one-way coupling assumption. For nano-particles in the size range of 5 to $200 \mathrm{~nm}$, in addition to the Brownian excitation, the electrostatic and gravitational forces were included in the analysis. Different charging mechanisms including field and diffusion charging as well as the Boltzmann charge distributions were investigated. The simulation methodology was first validated for Brownian and electrostatic forces. For the combined field and diffusion charging, the simulation results showed that in the presence of an electric field of $10 \mathrm{kV} / \mathrm{m}$, the electrostatic force dominates the Brownian effects. However, when the electric field was $1 \mathrm{kV} / \mathrm{m}$, the Brownian motion strongly affected the particle dispersion and deposition processes. For the electric field intensity of $1 \mathrm{kV} / \mathrm{m}$, for $10 \mathrm{~nm}$ and 100nm particles, the deposition efficiencies for the combined effects of electrostatic and Brownian motion were, respectively, about $27 \%$ and $161.2 \%$ higher than the case in the
\end{abstract}

\footnotetext{
* Corresponding author.

E-mail address: pouyan.talebizadehsardari@nottingham.ac.uk (P. Talebizadeh Sardari).
} 
absence of electric field. Furthermore, particles with the Boltzmann charge distribution had the maximum deposition for $20 \mathrm{~nm}$ particles.

Keywords: Nano-particles, Lagrangian particle tracking, Electrostatic force, Brownian diffusion, Deposition efficiency, Boltzmann charging, Diffusion and field charging.

\section{Introduction}

It is well-known that air pollutants have serious adverse health effects (Shy et al., 1978). Particulate matter (PM), especially nano-particles, can infiltrate deep into the human lung and can cause cancer and other lung diseases (Cohen \& Pope, 1995). Therefore, the development of air pollution control devices has attracted considerable attention in recent years (Talebizadeh et al., 2014). For PM removal from the emissions of various types of engines including air breathing engines, there are a number of devices such as filters, cyclones, nonthermal plasma and electrostatic precipitators (Babaie et al., 2015; Takasaki et al., 2015; Tian \& Ahmadi, 2007; Tu et al., 2012; Zheng et al., 2004; Zhou et al., 2017). Gas turbine engines are a source of PM emissions with diameters less than 2.5 microns, which are subject to regulation under the National Ambient Air Quality Standards (George et al., 1969). Effective pollution control of gas turbine engines has been a major concern in the design of modern aircraft propulsion systems (Schnelle et al., 2015).

Concerning nano-particles, Brownian diffusion is the main mechanism of particle dispersion and deposition (Guha, 2008; Talebizadeh et al., 2015; Zahmatkesh, 2008). The original model for simulating Brownian motion in the Lagrangian particle trajectory approach was introduced by Li and Ahmadi (1993a) and Ounis et al. (1993). Charged particles are affected by different electrostatic forces in the presence of an electric field (He \& Ahmadi, 1999; Kim et al., 2006; Malekian et al., 2018; Mayya et al., 2004). Therefore, the mechanisms of 
Brownian diffusion and electrophoresis are in competition for transport and deposition of charged nano-particles.

The deposition of charged particles in human airways during inhalation has been extensively studied, and it is shown that electrostatic forces increase particle deposition in human airways (Chan et al., 1978; Melandri et al., 1983). Aerosol particles naturally carry a certain number of electronic units of charge according to the Boltzmann charge distribution. When aerosols carry a high level of charge, the electrostatic forces significantly affect the particle deposition rate (Bailey et al., 1998). Cohen et al. (1998) compared the deposition of charged aerosol particles in hollow-cast models of human airways due to the image force. They showed that the deposition rate of $20 \mathrm{~nm}$ particles that carry one electronic unit of charge was 3.4 times that of the same size particle that carry the average of Boltzmann charge distribution and 5.3 times the neutral $20 \mathrm{~nm}$ particles. Furthermore, many studies on the useful effect of electrostatic forces on deposition of charged particles with the aim of targeted drug delivery were reported in the literature (Koullapis et al., 2016; Majid et al., 2016; Ruzer \& Harley, 2004).

Charged particle deposition and electrophoretic effects have been also studied extensively in other engineering applications including electrostatic precipitators and filters, particle separation devices and water purification (Besra \& Liu, 2007; Jaworek et al., 2018; Tsai et al., 2005). In the filters, the electrostatic charging has been used to increase the collection efficiency of particles (Jaworek et al., 2007). In electrostatic precipitators, the electrostatic force is the main mechanism for particle removal from the exhaust gas (He et al., 2017). Goo et al. (1997) studied particle deposition in a wire-plate electrostatic precipitator using the Lagrangian particle tracking method. Dixkens and Fissan (1999) studied particle deposition in electrostatic precipitators numerically and experimentally. They considered the effects of diffusion, electrostatic force, thermophoresis and sedimentation, and included the effects of 
field charging. Yu et al. (2017) studied deposition of submicron particles in an ionic air purifier experimentally ranging from $30 \mathrm{~nm}$ to $300 \mathrm{~nm}$ and showed higher deposition of nanoparticles with higher dielectric constant. Tu et al. $(2017,2018)$ investigated experimentally and numerically particle removal from exhaust flue gas in a novel electrostatic filter using perforated plates as the collecting electrodes. They showed that the openings change the electric field near the plate which improves the deposition efficiency of particles. Dong et al. (Dong et al., 2018) studied the charging and transport of fine particles in a wire-plate electrostatic precipitator using multiple wire electrodes. They employed the Lagrangian particle tracking method and considered the effects of Brownian diffusion and electrophoresis and analysed the influence of applied voltage.

In contrast to the Eulerian model which neglects the particle inertia effect, the Lagrangian approach tracks individual particle motions within the flow field considering different forces (Longest \& Xi, 2007). In the Lagrangian tracking method, particle deposition is assumed to occur upon wall contact. The Lagrangian approach, however, requires higher computational effort for simulating a large number of particles in order to accurately calculate the deposition rate (Longest et al,, 2004). The Eulerian approach avoids simulating a large number of particles since it deals directly with particle concentration; however, this approach requires significant modifications in order to consider the effects of inertial and electrophoresis forces (Wang, Flagan, \& Seinfeld, 2002).

In this study, the Lagrangian particle tracking method is used for evaluating the transport and deposition of nano-particles in fully developed laminar duct flow, considering the effects of Brownian, electrostatic and gravity forces. Different mechanisms of field and diffusion charging as well as the Boltzmann charge distribution are considered. A comparative study of the effects of electrophoresis and Brownian diffusion on charged nano-particle deposition in the presence of different electric fields is performed. 


\section{CFD modelling and boundary conditions}

\subsection{Fluid flow modelling}

For 2D incompressible and steady airflow between two parallel plates, the exact solution for fully developed laminar velocity profile is given as:

$u^{f}(y)=\frac{3}{2} u_{a v}\left(1-\frac{y^{2}}{H^{2}}\right)$

Here the width and length of the duct used in this study are, respectively, $2 \mathrm{~mm}(\mathrm{H}=1 \mathrm{~mm})$ and $4 \mathrm{~cm}$. Note that $u_{a v}$ is the mean flow velocity which is assumed to be $1 \mathrm{~m} / \mathrm{s}$, which results in the flow Reynolds number of about 130. Since the flow is considered fully developed, Eq. (1) is used throughout the domain. For airflow, it is assumed that the flow is not affected by the presence of electric field. The schematic of the laminar duct flow is shown in Fig. 1

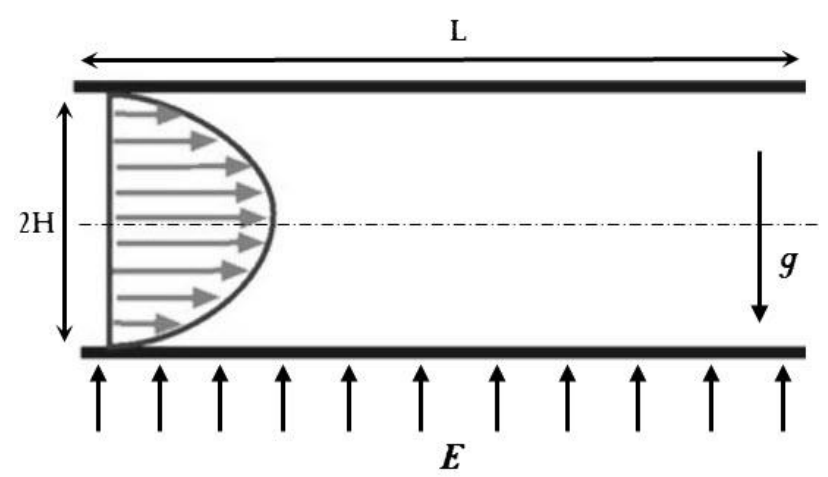

Fig. 1. Schematic of the fully developed airflow between two parallel plates.

\subsection{Particle equation of motion}

The trajectories of nano-particles with diameters 5, 10, 20, 40, 100 and $200 \mathrm{~nm}$ are evaluated using the Lagrangian particle tracking method under the assumption of one-way coupling 
(Abouali \& Ahmadi, 2005). Fluid drag force is always present and balances with the inertia, Brownian excitation and/or electrostatic forces. Due to the range of nano-particle diameters studied, the Brownian force should be included in the analysis. The electrostatic force is also included due the presence of an applied electric field across the duct. The effect of gravity is also considered for completeness. Therefore, the appropriate equation governing the motion of a spherical particle is expressed as (Li \& Ahmadi, 1992a):

$\frac{u_{i}^{p}}{d t}=F_{i}^{D}+F_{i}^{B}+\frac{F_{i}^{e}}{m}+F_{i}^{g}$

Here $F_{i}^{D}$ is the drag force given as:

$F_{i}^{D}=\frac{C_{D} R e_{P}\left(u_{i}^{f}-u_{i}^{p}\right)}{24 \tau}$

where $u_{i}^{p}$ and $u_{i}^{f}$ are, respectively, the components of the particle and the local fluid velocity.

The x-component of fluid velocity is given by Eq. (1) with the y-component being zero for the fully developed flow conditions considered in this study. In Eq. (3) $R e_{P}$ is the particle Reynolds number defined as $R e_{P}=\frac{\mu_{f}\left|\mathbf{u}^{f}-\mathbf{u}^{p}\right| d_{p}}{\rho_{f}}$, and $\tau$ is the particle relaxation time given by $\tau=\frac{d_{p}{ }^{2} \rho_{p} C_{c}}{18 \mu_{f}}$, where $d_{p}$ is the particle diameter, $\mu_{f}$ and $\rho_{f}$ are, respectively, the fluid viscosity and density, and $\rho_{p}$ is the particle density. Here $C_{c}$ is the Cunningham correction factor to the Stokes drag calculated as (Hinds, 2012):

$C_{c}=1+\frac{2 \lambda}{d_{p}}\left(1.257+0.4 e^{-\left(1.1 d_{p} / 2 \lambda\right)}\right)$

Where $\lambda$ is the mean free path of air which is equal to $65 \mathrm{~nm}$ under normal condition.

In Eq. (3), $C_{D}$ is the drag coefficient defined as:

$\begin{array}{ll}C_{D}=\frac{24}{R e_{p}} & \text { if } R e_{p}<1 \\ C_{D}=\frac{24\left(1+15\left(R e_{p}{ }^{0.687}\right)\right)}{R e_{p}} & \text { if } R e_{p}>1\end{array}$

The amplitude of the Brownian force is given as (Li \& Ahmadi, 1992a; Ounis et al., 1993): 
$F^{B}=\zeta \sqrt{\frac{\pi S_{0}}{\Delta t}}$

where $\zeta$ is a zero-mean, unit-variance independent Gaussian random number, $\Delta t$ is the timestep for particle integration and $S_{0}$ is a spectral intensity function defined as (Li \& Ahmadi, 1992a),

$S_{0}=\frac{216 v k_{B} T}{\pi^{2} \rho_{g} d_{p}{ }^{2}\left(\frac{\rho_{p}}{\rho_{f}}\right)^{2} C_{c}}$

Here $T$ is the absolute temperature of the fluid, $v$ is the kinematic viscosity and $k_{B}$ is the Boltzmann constant.

Four electrostatic forces act on a charged particle near a conducting surface. These are Coulomb, image, dipole-dipole and dielectrophoretic forces. He and Ahmadi (1999) showed that the dipole-dipole and dielectrophoretic forces are typically much lower than Coulomb and image forces. In the presence of an electric field, the Coulomb force is typically the dominant force. The image force is present in the absence of an electric field near a conducting surface. In this study, both Coulomb and image forces are considered and the total electrostatic force is given as (He \& Ahmadi, 1999; Hinds, 2012):

$F^{e}=F^{C}+F^{i}$

$F^{C}$ is the coulomb force given as

$F^{C}=q E$

where $E$ is the electric field intensity and $q$ is the amount of charge on particles given as

$q=n e$

where $n$ is the number of electrical charges on a particle and $e$ is the electric charge unit equal to $1.602 \times 10^{-19} \mathrm{C}$. Note that the direction of the Coulomb force is related to the electric field which can be toward or away from the wall.

$F^{i}$ is the image force given as: 
$F^{i}=\frac{q^{2}}{16 \pi \varepsilon_{0} y_{c}^{2}}$

where $\varepsilon_{0}$ is electric permittivity of vacuum which is equal $8.859 \times 10^{-12} \mathrm{~F} / \mathrm{m}$ and $y_{c}$ is the distance between the particle centre and the wall.

In a neutral atmosphere, aerosols generally carry the Boltzmann charge distribution. Accordingly, the fraction that has $n$ electronic units of charge is given by:

$f(n)=\frac{\exp \left(-K_{E} n^{2} e^{2} / d_{p} k_{B} T\right)}{\sum_{n=-\infty}^{+\infty} \exp \left(-K_{E} n^{2} e^{2} / d_{p} k_{B} T\right)}$

where $K_{E}=\frac{1}{4 \pi \varepsilon_{0}}$ is a constant of proportionality that depends on the system of units used. Here $K_{E}=9 \times 10^{9} \mathrm{Nm}^{2} / \mathrm{C}^{2}$ for SI units (Hinds, 2012).

Using (12), the average number of absolute charge carried on a particle is given as (He \& Ahmadi, 1999):

$|\bar{n}|=\sum_{n=0}^{\infty}|n| f(|n|)$

The average number of positive or negative charge then is $|n| / 2$.

Aerosol particles are also charged by field and diffusion charging amongst other mechanisms (Hinds, 2012). In the presence of an electric field, the particles are charged due to the impact of unipolar ions which is called field charging. This mechanism is more pronounced for particles with diameter greater than 1 micron. The maximum number of charges on a particle under field charging, which is achieved under equilibrium conditions, is given as (Hinds, 2012),

$n=\left(\frac{3 \varepsilon_{D 0}}{\varepsilon_{D 0}+2}\right) \frac{E d_{p}^{2}}{4 K_{E} e}$

where $\varepsilon_{D 0}$ is the dielectric constant of the particles.

Diffusion charging results from the accidental impacts between the particles and bipolar ions due to Brownian motion. For particles with the diameter less than 1 micron, diffusion charging is the main mechanism for particle charging, which is given as (Hinds, 2012), 
$n=\frac{d_{p} k T}{2 K_{E} e^{2}} \ln \left[1+\frac{\pi K_{E} d_{p} \overline{c_{l}} e^{2} N_{i} t}{2 k T}\right]$

where $\bar{c}_{i}$ is the thermal mean velocity of ions equal to $2.4 \times 10^{10} \mathrm{~cm} / \mathrm{s}$ and $N_{i}$ is the concentration of ions. In this study, the usual value of $N_{i} t=10^{8}$ ion. $\mathrm{s} / \mathrm{cm}^{3}$ is used (He \& Ahmadi, 1999).

In Equation (2) $F_{i}^{g}$ is the effective acceleration of gravity given as

$F_{i}^{g}=\frac{g_{i}\left(\rho_{p}-\rho_{f}\right)}{\rho_{p}}$

Note that $F_{g}$ is included in the simulation of particle deposition in the present study, however, the effect of gravity on deposition of nano-particles smaller than $200 \mathrm{~nm}$ is negligible (Talebizadeh, Rahimzadeh, Ahmadi, Brown, \& Inthavong, 2016).

In this paper, the deposition velocity and deposition efficiency are evaluated. Deposition velocity is defined as the flux of nano-particles to the wall per unit concentration per unit time. For calculating the deposition velocity in Lagrangian particle tracking approach, for a uniform initial injection of $N_{0}$ particles distributed in a region within a distance of $H_{0}=30$ wall units from the wall, the deposition velocity is given as (Li \& Ahmadi, 1993b),

$u_{d}=\frac{N_{d} / t_{d}}{N_{0} / H_{0}}$

where $N_{d}$ is the number of deposited particles in the time duration $t_{d}$. Note that the wall unit is defined as $v / u^{*}$. In practice of the simulation, the time duration should be long enough to obtain a quasi-equilibrium condition where the rate of the total number of deposited particles reaches to a constant value (He \& Ahmadi, 1999). The non-dimensional form of the deposition velocity is given by

$u_{d}^{+}=\frac{u_{d}}{u^{*}}$

where $u^{*}$ is the shear velocity defined as 
$u^{*}=\sqrt{\frac{\overline{\tau_{w}}}{\rho}}$

where $\overline{\tau_{w}}$ is the wall shear stress.

To calculate the deposition efficiency in a fully developed flow, at the injection plane, the inlet particle flux profile was generated using the velocity profiles given by Eq. (1) to generate appropriate mass flux for each particle size. Then, the deposition efficiency is calculated based on the ratio of mass deposition rate on the wall to the inlet mass flow rate of particles as (Longest \& Xi, 2007):

$D E=\frac{\dot{m}_{w}}{\dot{m}_{\text {in }}}$

An in-house FORTRAN code was developed for solving the particle equation of motion and for evaluating the deposition efficiency, as well as deposition velocity. The particle equation of motion is integrated in time using an appropriate time step for a fixed flow domain. 10000 particles are used for the evaluation of the deposition velocity and deposition efficiency. This number was determined by simulations with less and more particles that was shown to have negligible effect on the deposition results. Note that in the present simulations, the direction of the electrostatic force is upward while the gravity is downward. Since the objective of this study is to provide an understanding of the contributions of different forces including the electrostatic force along with the Brownian diffusion effects, a simple 2D duct is used.

\section{Results and discussion}

Table 1 lists the amount of charges (electronic units of charge) for different particle diameters and various charging mechanisms. For nano-particles, which are of concern in this study, the contributions of diffusion charging are higher than those by field charging for all the studied electric field intensities. Furthermore, by increasing the electric field intensity from $1 \mathrm{kV} / \mathrm{m}$ to $100 \mathrm{kV} / \mathrm{m}$, the contribution of field charging increases. For smaller particles however, the 
amount of field charging is still negligibly small even for field intensity of $100 \mathrm{kV} / \mathrm{m}$. For the Boltzmann charging distribution, the number of charge increases with the diameter of particles, but it is much smaller than those of diffusion and field charging. Note that the temperature of the fluid and particles is assumed to be fixed at $288 \mathrm{~K}$.

Table 1. The number of electronic units of charge carried by particles of different diameters for various charging mechanisms and electric field intensities.

\begin{tabular}{|c|c|c|c|c|c|c|}
\hline Dia. (nm) & Boltzmann & Diff. & Field & Diff.+Field & Field & Diff.+Field \\
\cline { 4 - 7 } & $|\bar{n}|$ & & \multicolumn{2}{|c|}{$\mathrm{E}=1 \mathrm{kV} / \mathrm{m}$} & \multicolumn{2}{|c|}{$\mathrm{E}=100 \mathrm{kV} / \mathrm{m}$} \\
\hline 5 & 0.00002 & 0.107 & $9.3 \mathrm{E}-06$ & 0.107 & $9.3 \mathrm{E}-04$ & 0.108 \\
\hline 10 & 0.00604 & 0.27 & $3.72 \mathrm{E}-05$ & 0.27 & $3.72 \mathrm{E}-03$ & 0.274 \\
\hline 20 & 0.09930 & 0.656 & $1.49 \mathrm{E}-04$ & 0.656 & $1.49 \mathrm{E}-02$ & 0.6709 \\
\hline 40 & 0.32640 & 1.546 & $5.95 \mathrm{E}-04$ & 1.547 & $5.95 \mathrm{E}-02$ & 1.606 \\
\hline 100 & 0.66452 & 4.65 & $3.72 \mathrm{E}-03$ & 4.654 & 0.372 & 5.022 \\
\hline 200 & 0.99573 & 10.49 & $1.49 \mathrm{E}-02$ & 10.51 & 1.49 & 11.98 \\
\hline
\end{tabular}

Table 2 presents the amount of charge units per unit mass of a particle which provides important information for interpreting the electrophoresis effects. Note that $\rho_{f}=$ $1.225 \mathrm{~kg} / \mathrm{m}^{3}$ and $\rho_{p}=2000 \times \rho_{f}$ (for carbon particles) is assumed. It is seen that for diffusion and field charging, the number of charge per unit mass decreases as particle diameter increases. Also diffusion charging makes the bulk of the contributions to the charging of these ultrafine particles. For the Boltzmann charge distribution, the maximum number of charge per unit mass occurs for $20 \mathrm{~nm}$ particles.

Table 2. The number of charge per unit mass (gr) for different particle diameters and for various charging mechanisms and electric field intensities.

(The unit is electronic unit of charge/gr.) 


\begin{tabular}{|c|c|c|c|c|c|c|}
\hline Dim (nm) & Boltzmann & Diff. & Field & Diff.+Field & Field & Diff.+Field \\
\cline { 4 - 7 } & $|\bar{n}|$ & & \multicolumn{2}{|c|}{$\mathrm{E}=1 \mathrm{kV} / \mathrm{m}$} & \multicolumn{2}{|c|}{$\mathrm{E}=100 \mathrm{kV} / \mathrm{m}$} \\
\hline 5 & $1.36 \mathrm{E}+17$ & $7.27 \mathrm{E}+20$ & $6.32 \mathrm{E}+16$ & $7.27 \mathrm{E}+20$ & $6.32 \mathrm{E}+18$ & $7.33 \mathrm{E}+20$ \\
\hline 10 & $5.13 \mathrm{E}+18$ & $2.29 \mathrm{E}+20$ & $3.16 \mathrm{E}+16$ & $2.29 \mathrm{E}+20$ & $3.16 \mathrm{E}+18$ & $2.33 \mathrm{E}+20$ \\
\hline 20 & $1.05 \mathrm{E}+19$ & $6.96 \mathrm{E}+19$ & $1.58 \mathrm{E}+16$ & $6.96 \mathrm{E}+19$ & $1.58 \mathrm{E}+18$ & $7.12 \mathrm{E}+19$ \\
\hline 40 & $4.33 \mathrm{E}+18$ & $2.05 \mathrm{E}+19$ & $7.89 \mathrm{E}+15$ & $2.05 \mathrm{E}+19$ & $7.89 \mathrm{E}+17$ & $2.13 \mathrm{E}+19$ \\
\hline 100 & $5.64 \mathrm{E}+17$ & $3.95 \mathrm{E}+18$ & $3.16 \mathrm{E}+15$ & $3.95 \mathrm{E}+18$ & $3.16 \mathrm{E}+17$ & $4.26 \mathrm{E}+18$ \\
\hline 200 & $1.06 \mathrm{E}+17$ & $1.11 \mathrm{E}+18$ & $1.58 \mathrm{E}+15$ & $1.12 \mathrm{E}+18$ & $1.58 \mathrm{E}+17$ & $1.27 \mathrm{E}+18$ \\
\hline
\end{tabular}

Tables 1 and 2 show that for the range of nano-particles studied here, the diffusion charging is dominant and the summation of diffusion and field charging does not vary too much for different electric field intensities. However, the influence of electric field intensity is quite significant due to increase of Coulomb force. These issues are further discussed in Section 3.2.

\subsection{Validations}

In the absence of other forces for a constant airflow velocity, the variance of particle location under Brownian excitation is given as (Einstein, 1956; Li \& Ahmadi, 1992b):

$\sigma_{y}^{2}(t)=2 D T$

Where $D=\frac{k T C_{c}}{3 \pi \mu d_{p}}$ is the particle diffusivity. Fig. 2 compares the simulated variance of $10 \mathrm{~nm}$ particles with prediction of Eq. (19). Here 1000 particles are released from the centre of the duct. It is seen that simulation results are in excellent agreement with the theoretical model. Note that the size of the time step is considered 0.5 micro-second for the simulations and the results are not changed with reducing the time step size. 


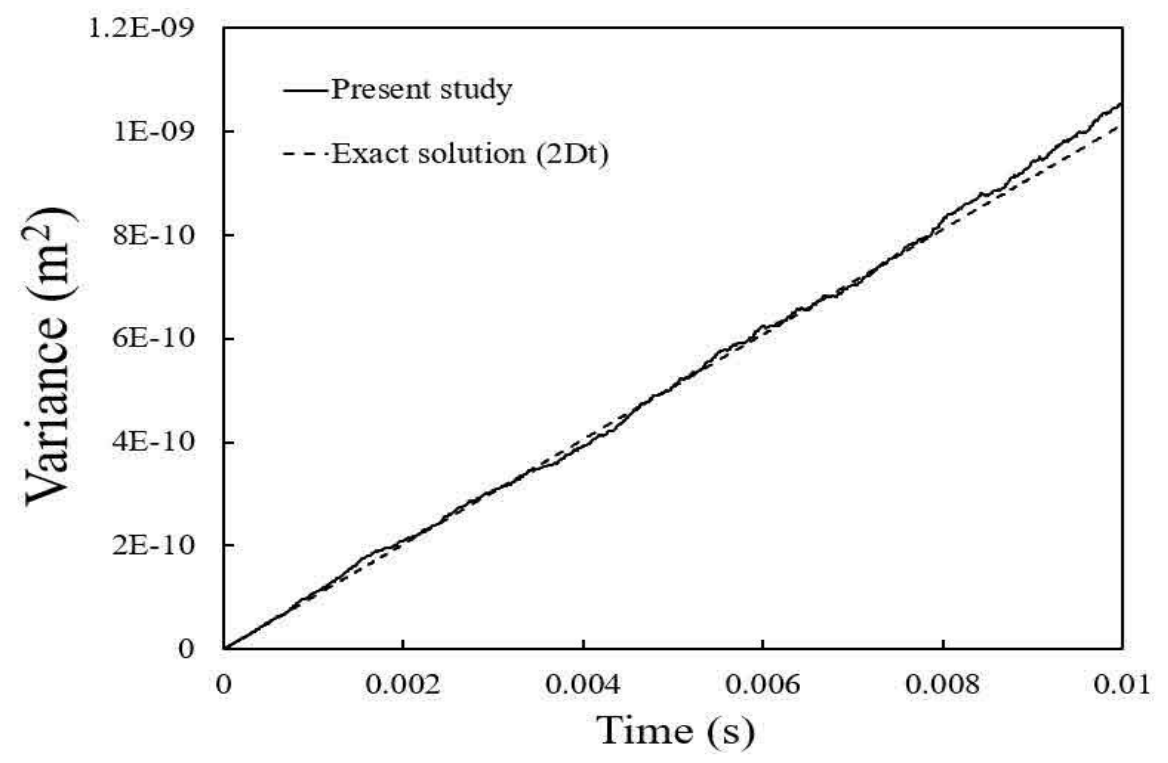

Fig. 2. Comparison of predicted variance of $10 \mathrm{~nm}$ particle position with the exact solution.

For verifying the results for Brownian deposition in a fully developed duct flow, the simulation results are compared with the analytical expression reported in the literature. Ingham (1976) determined the deposition efficiency by solving the concentration equation considering sedimentation for fully developed duct flows as:

$D E=2.42264 s^{2 / 3}-0.3 s-\left(0.08608-1.33909 \sigma_{s}^{2}\right) s^{4 / 3}$

where

$s=\frac{2 D L}{3 Q h}$

where $Q$ is the air flow rate, $h$ is the duct half-width and $L$ is the duct length. $\sigma_{S}$ is the sedimentation coefficient, which was zero in this study. The Gormley and Kennedy (1948) expression is given as,

$D E=2.44164 s^{2 / 3}-0.3 s-0.0772 s^{4 / 3}$

A series of simulations for particle deposition in a $4 \mathrm{~cm}$ long and $2 \mathrm{~mm}$ wide duct under fully developed laminar flow condition with the mean velocity of $1 \mathrm{~m} / \mathrm{s}$ was performed. Fig. 3 compares the predicted variation of deposition efficiency for different particle diameters subject to Brownian excitation with the expressions of Ingham (1976) and Gormley and 
Kennedy (1948) given by (21) and (23). This figure shows that the present results are in excellent agreement with the analytical models.

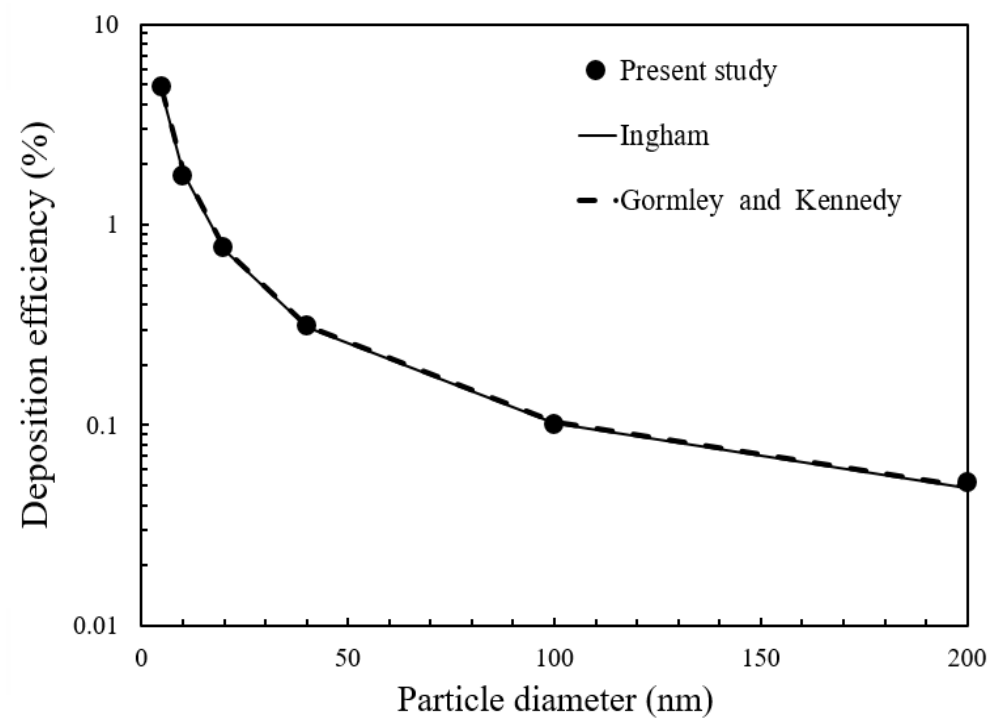

Fig. 3. Comparison of the predicted deposition efficiency for different particle diameters with analytical expressions.

For verifying the computational model for electrical deposition a series of simulations in the absence of Brownian effects was performed. The corresponding non-dimensional deposition velocity as computed from Eqs. (15) and (16) are compared with the expression for the electrical terminal velocity given as (He \& Ahmadi, 1999; Hinds, 2012):

$u_{e}^{+}=\frac{q E C_{c}}{3 \pi \mu d u^{*}}$

For the electric field intensities of 10 and $100 \mathrm{kV} / \mathrm{m}$, Figs. 4 compares the simulation results in the absence of Brownian motion according to Eq. (15) with the prediction of Eq. (24). Here a mean fluid velocity of $1 \mathrm{~m} / \mathrm{s}$ was assumed. It is seen that the simulation results are in excellent agreement with Eq. (24). 


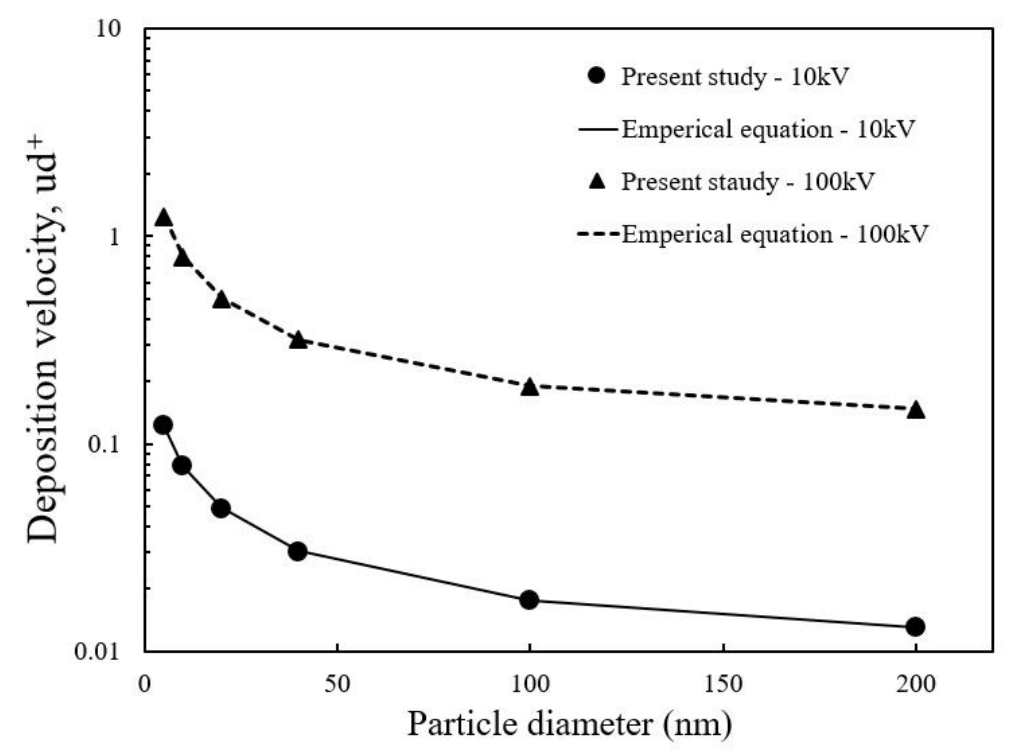

Fig. 4. Comparison of the predicted variation of deposition velocity for different particle diameters in the absence of Brownian effects for the electric field intensity of $10 \mathrm{kV} / \mathrm{m}$ and $100 \mathrm{kV} / \mathrm{m}$, for a mean velocity of $1 \mathrm{~m} / \mathrm{s}$.

\subsection{Effects of various forces}

Fig. 5 shows the deposition velocity for the electric field intensity of $10 \mathrm{kV} / \mathrm{m}$ considering both electrostatic and Brownian forces. Here the combined diffusion and field charging for the particles is assumed and average flow velocity is $1 \mathrm{~m} / \mathrm{s}$. It is seen that the effect of electrostatic force for combined field and diffusion charging is much higher than that of the Brownian diffusion for all particle diameters. In addition, the deposition velocity decreases as particle size increases. This observation is consistent with the results shown in Table 2 that the number of charges per unit mass of the particle for diffusion and field charging decreases as particle size increases. 


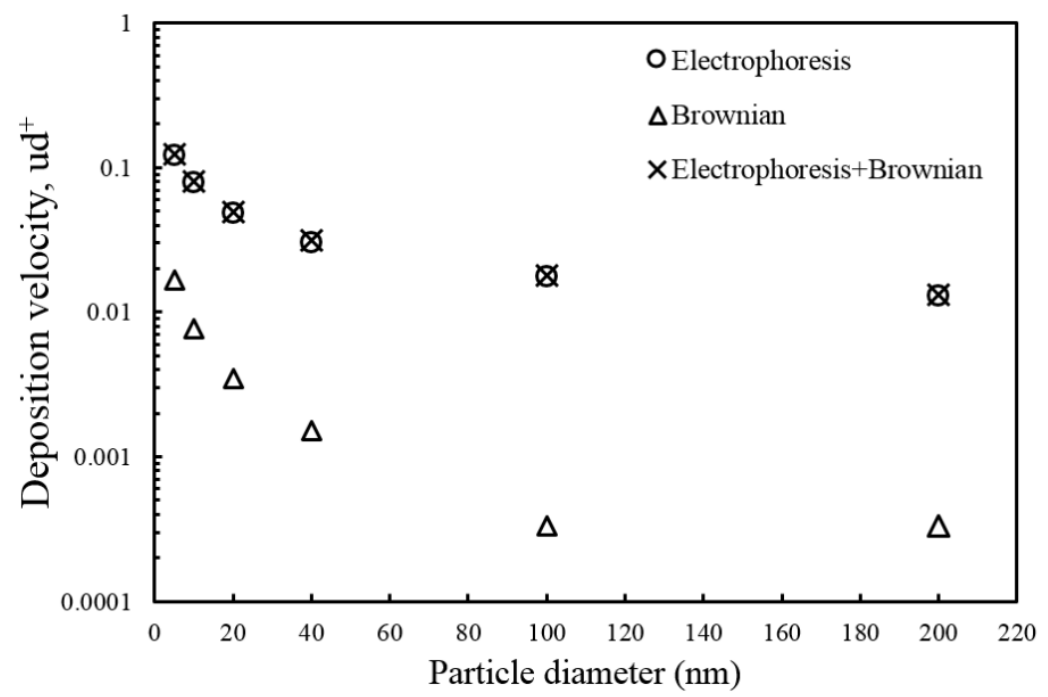

Fig. 5. The effect of various forces on deposition velocity for different particle diameters for an electric field of $10 \mathrm{kV} / \mathrm{m}$.

Fig. 6 displays the particle deposition efficiencies in a fully developed duct flow with the mean velocity of $1 \mathrm{~m} / \mathrm{s}$ in the presence of an electric field intensity of $1 \mathrm{kV} / \mathrm{m}$. Here the particles are assumed to carry the number of charges under the combined diffusion and field changing. For this low intensity of electric field, it is seen that for $5 \mathrm{~nm}$ particles, the Brownian excitation is the dominant mechanism for particle deposition. However, for larger 100 and $200 \mathrm{~nm}$ particles, the Brownian effects decreases and the electrostatic precipitation become dominant. Fig. 6 also shows that the slope of the variation of Brownian deposition (in the absence of electrostatic effects) is higher than electrostatic deposition (in the absence of Brownian effects). That is, as expected the Brownian effect is a more sensitive function of particle diameter compared to the electrostatic effect. This figure also shows that for nanoparticles carrying charges according to diffusion and field charging, both the electrophoresis and Brownian diffusion contribute to the deposition process. For certain ranges of particle diameters, the effect of electrostatic force dominates, while for very small sizes the Brownian effects become dominant. 


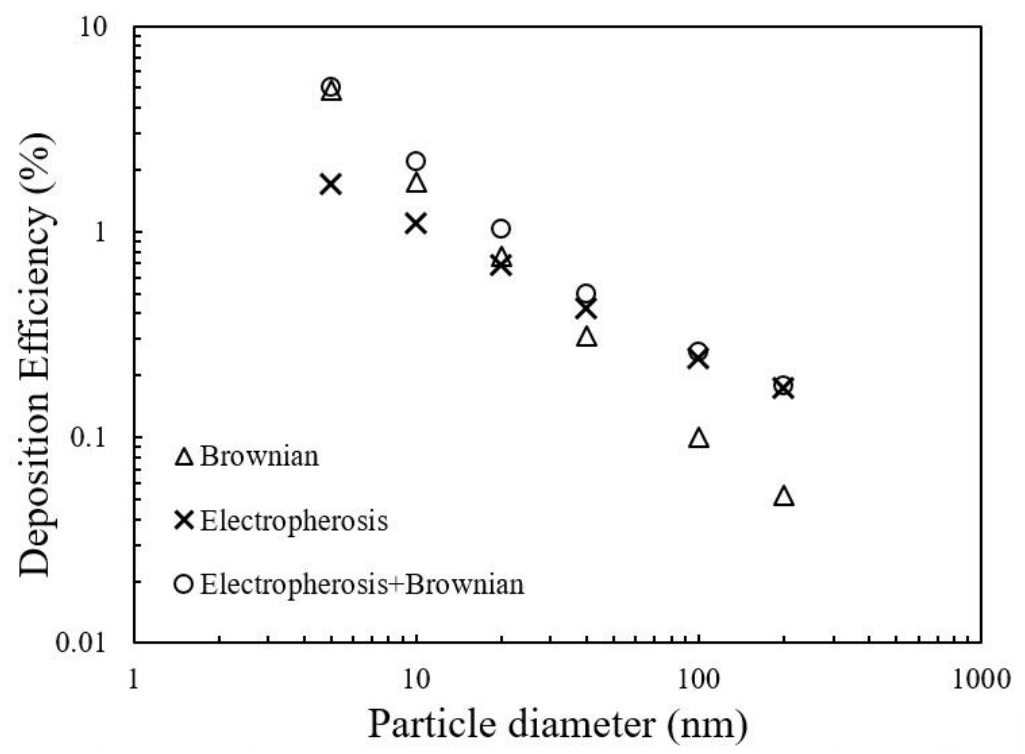

Fig. 6. Variations of deposition efficiency as a function of particle diameter for an electric field intensity of $1 \mathrm{kV} / \mathrm{m}$ for different forces.

Fig. 7 shows the variation of deposition efficiency for various particle diameters for a fully developed laminar flow in the presence of an electric field of $10 \mathrm{kV} / \mathrm{m}$. The particles are charged by the combined diffusion and field changing. It is seen that the deposition efficiency decreases as particle size increases. For this high electric field intensity the electrostatic sedimentation makes the bulk of contribution to the deposition velocity and the effect of Brownian diffusion is negligibly small. 


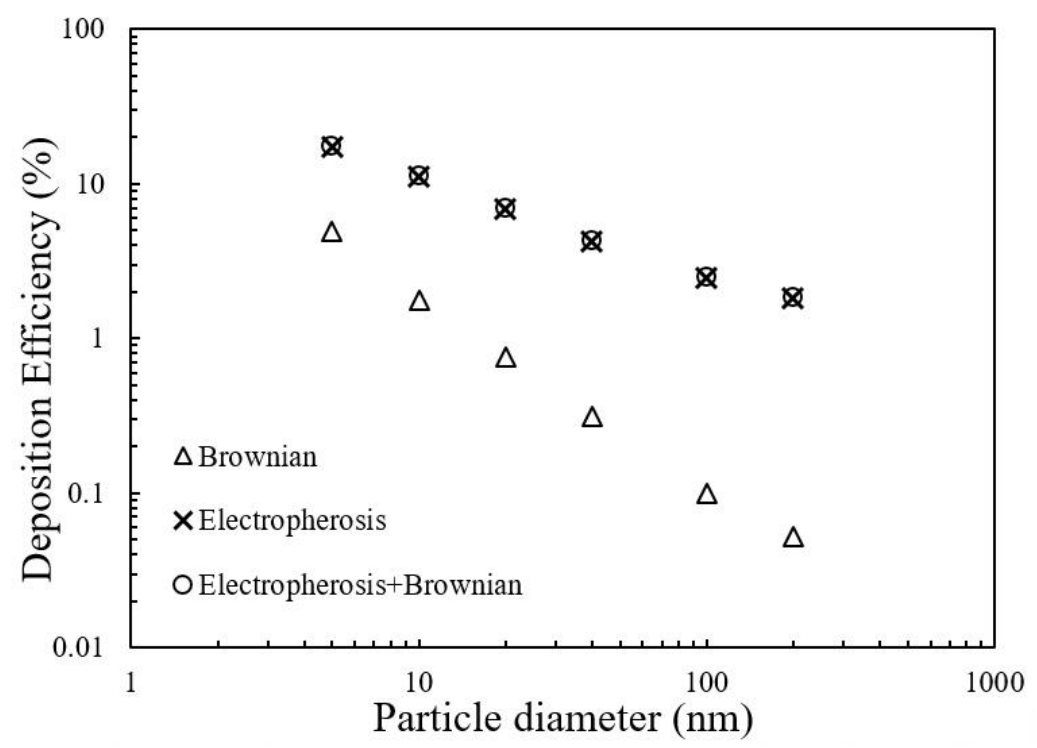

Fig. 7. Variations of deposition efficiency as a function of particle diameter for an electric field intensity of $10 \mathrm{kV} / \mathrm{m}$.

Fig. 8 shows the time variations of number of deposited and escaped particles as well as the deposition efficiency for $5 \mathrm{~nm}$ particles considering both Brownian and electrostatic effects for an electric field intensity of $10 \mathrm{kV} / \mathrm{m}$. It is seen that after $0.027 \mathrm{~s}$ from the start of simulations, the particles begin to escape from the outlet. Furthermore, after around $0.1 \mathrm{~s}$ all particles that are introduced at the inlet are deposited or escaped from the domain. It can be seen that after $0.06 \mathrm{~s}(60 \%$ of the simulation time) almost all particles exit from the solution domain and the deposition efficiency becomes constant. Furthermore, it is seen that there is a transient region at the beginning as the rate of particle deposition develops. After the initial stages, the rate of particle deposition is roughly constant until about $0.05 \mathrm{~s}$. In the final stage, the deposition efficiency is constant and the rate of deposition approaches zero as the majority of injected particles are either deposited or escaped. Understanding of the deposition behaviour of particles is useful for optimization of particle removal devices. 


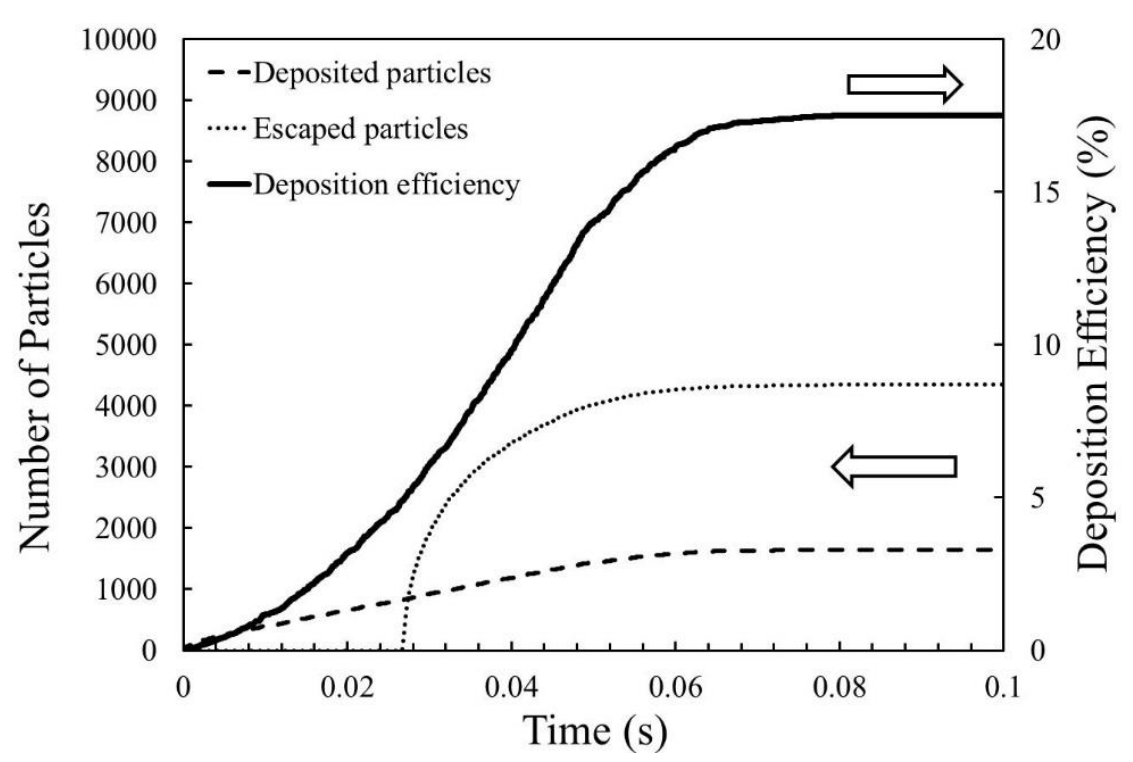

Fig. 8. Variations of the number of deposited and escaped particles as well as deposition efficiency for $5 \mathrm{~nm}$ particles with diffusion/field charging versus time for $\mathrm{E}=10 \mathrm{kV} / \mathrm{m}$.

Fig. 9 illustrates the variation of deposition efficiency as a function of particle diameters for the Boltzmann charge distribution in the presence of a $10 \mathrm{kV} / \mathrm{m}$ electric field. In the discussion of Table 2, the number of charges per unit mass was observed to increase with size from $5 \mathrm{~nm}$ to $20 \mathrm{~nm}$ particles and then decreases by further increase of particle diameter. Only considering the number of charges is insufficient to assess the behaviour of nano-particles in the presence of an electric field. Therefore, when the electrostatic force alone is considered, the deposition efficiency reaches to its maximum for $20 \mathrm{~nm}$ particles. The Brownian diffusion effects increase as particle size decreases. When both Brownian and electrostatic effects are included, for the Boltzmann charge distribution, for particles less than 20nm the Brownian diffusion is dominant and the effect of electrophoresis is almost negligible. For 40nm particles and larger, the electrostatic force becomes more dominant; however, the effect of Brownian diffusion cannot be neglected. 


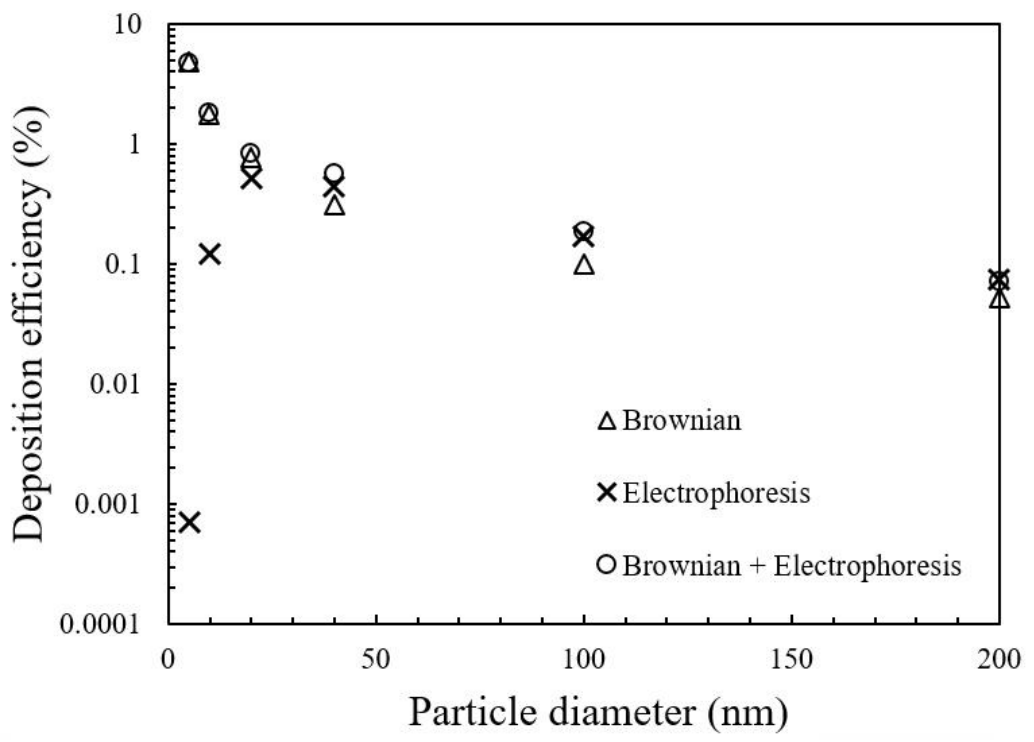

Fig. 9. The variation of deposition efficiency as a function of particle diameter for the Boltzmann charge distribution for $\mathrm{E}=10 \mathrm{kV} / \mathrm{m}$.

\section{Conclusions}

In this paper, the effects of Brownian diffusion on nano-particle deposition in a fully developed duct flow were compared with electrophoresis in the presence of various electric field intensities. Different charging mechanisms including diffusion and field as well as the Boltzmann charge distribution were included in the analysis. For diffusion/field charging in the presence of a high intensity electric field, the presented results showed that the electrostatic forces dominate and the effect of Brownian diffusion on deposition fraction is comparatively negligibly small. However, for low electric fields, the Brownian effect is larger than the electrostatic effect, especially for particles smaller than $100 \mathrm{~nm}$.

For an electric field of $1 \mathrm{kV} / \mathrm{m}$ and the combined diffusion and field charging mechanism, the deposition efficiency of $10 \mathrm{~nm}$ and $100 \mathrm{~nm}$ nano-particles for the case that both electrostatic and Brownian effects were considered are, respectively, about $24.5 \%$ and $161.2 \%$ higher than the case when only Brownian diffusion was included. That is, the presence of an even low intensity electric field could cause a significant increase in the particle deposition efficiency. 
For the Boltzmann charge distribution, the electrostatic force effects peaked for the $20 \mathrm{~nm}$ particles.

The results of this study could find application for providing a guideline for evaluating the nano-particle transport and deposition in the presence of an electric field in devices such as pollution control devices, electrostatic precipitators, filters, particle separation equipment, monitoring devices, copier, printers and water purification devices.

\section{Nomenclature}

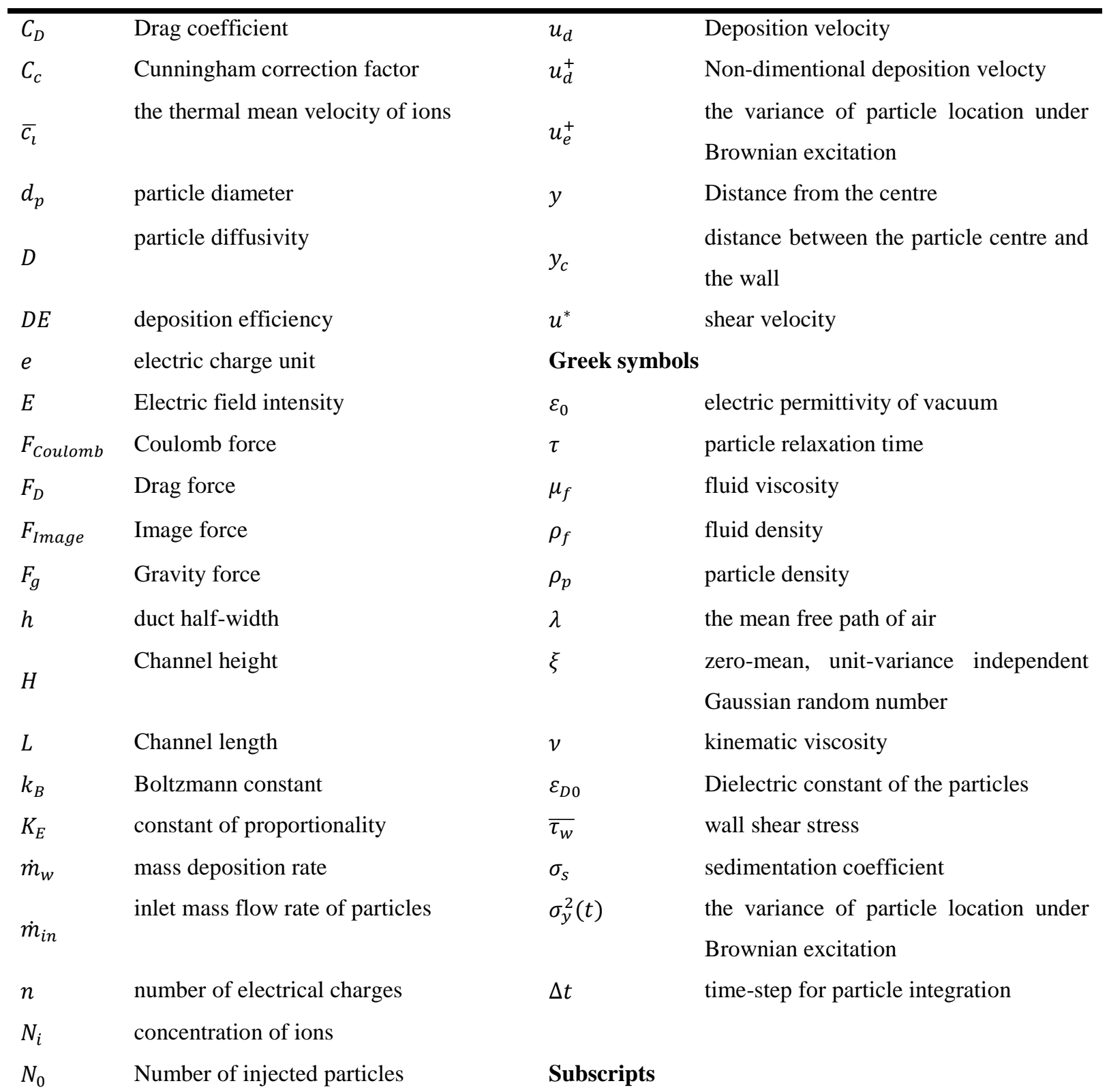




\begin{tabular}{llll}
$N_{d}$ & number of deposited particles & av & average \\
$q$ & the amount of charge on particles & $\mathrm{c}$ & Centre \\
$Q$ & air flow rate & $D$ & Drag \\
$R e_{p}$ & particle Reynolds number & $f$ & Fluid \\
$S_{0}$ & spectral intensity function & $P$ & Particle \\
$T$ & absolute temperature & & \\
$t_{d}$ & time duration & Superscripts & \\
$u$ & velocity & - & Mean value \\
$u_{a v}$ & Mean fluid velocity & & \\
\hline
\end{tabular}

\section{References}

Abouali, O., \& Ahmadi, G. (2005). A model for supersonic and hypersonic impactors for nanoparticles. Journal of Nanoparticle Research, 7(1), 75-88. doi: 10.1007/s11051-0047910-3

Babaie, M., Davari, P., Talebizadeh, P., Zare, F., Rahimzadeh, H., Ristovski, Z., \& Brown, R. (2015). Performance evaluation of non-thermal plasma on particulate matter, ozone and CO 2 correlation for diesel exhaust emission reduction. Chemical Engineering Journal, 276(15), 240-248.

Bailey, A. G., Hashish, A. H., \& Williams, T. J. (1998). Drug delivery by inhalation of charged particles. Journal of Electrostatics, 44(1), 3-10. doi: http://dx.doi.org/10.1016/S0304-3886(98)00017-5

Besra, L., \& Liu, M. (2007). A review on fundamentals and applications of electrophoretic deposition (EPD). Progress in materials science, 52(1), 1-61.

Chan, T. L., Lippmann, M., Cohen, V. R., \& Schlesinger, R. B. (1978). Effect of electrostatic charges on particle deposition in a hollow cast of the human larynx-tracheobronchial tree. Journal of Aerosol Science, 9(5), 463-468. doi: http://dx.doi.org/10.1016/0021$\underline{8502(78) 90009-5}$ 
Cohen, A. J., \& Pope 3rd, C. (1995). Lung cancer and air pollution. Environmental Health Perspectives, 103(Suppl 8), 219.

Cohen, B. S., Xiong, J. Q., Fang, C.-P., \& Li, W. (1998). Deposition of charged particles on lung airways. Health Physics, 74(5), 554-560.

Dixkens, J., \& Fissan, H. (1999). Development of an electrostatic precipitator for off-line particle analysis. Aerosol Science \& Technology, 30(5), 438-453.

Dong, M., Zhou, F., Zhang, Y., Shang, Y., \& Li, S. (2018). Numerical study on fine-particle charging and transport behaviour in electrostatic precipitators. Powder Technology, 330, 210-218. doi: https://doi.org/10.1016/j.powtec.2018.02.038

Einstein, A. (1956). Investigations on the Theory of the Brownian Movement: Dover Publications.

George, R. E., Verssen, J. A., \& Chass, R. L. (1969). Jet aircraft: A growing pollution source. Journal of the Air Pollution Control Association, 19(11), 847-855.

Goo, J. H., \& Lee, J. W. (1997). Stochastic simulation of particle charging and collection characteristics for a wire-plate electrostatic precipitator of short length. Journal of Aerosol Science, 28(5), 875-893. doi: http://dx.doi.org/10.1016/S0021-8502(96)00475-2

Gormley, P. G., \& Kennedy, M. (1948). Diffusion from a Stream Flowing through a Cylindrical Tube. Proceedings of the Royal Irish Academy. Section A: Mathematical and Physical Sciences, 52, 163-169. doi: 10.2307/20488498

Guha, A. (2008). Transport and deposition of particles in turbulent and laminar flow. Annu. Rev. Fluid Mech., 40, 311-341.

He, C., \& Ahmadi, G. (1999). Particle deposition in a nearly developed turbulent duct flow with electrophoresis. Journal of Aerosol Science, 30(6), 739-758. doi: 10.1016/S00218502(98)00760-5 
He, Z., Dass, E. T. M., \& Karthik, G. (2017). Design of electrostatic precipitator to remove suspended micro particulate matter from gas turbine inlet airflow: Part I. Experimental study. Journal of Aerosol Science, 108, 14-28. doi: https://doi.org/10.1016/j.jaerosci.2017.03.003

Hinds, W. C. (2012). Aerosol Technology: Properties, Behavior, and Measurement of Airborne Particles: Wiley.

Ingham, D. B. (1976). Simultaneous diffusion and sedimentation of aerosol particles in rectangular tubes. Journal of Aerosol Science, 7(5), 373-380. doi: 10.1016/00218502(76)90023-9

Jaworek, A., Krupa, A., \& Czech, T. (2007). Modern electrostatic devices and methods for exhaust gas cleaning: A brief review. Journal of Electrostatics, 65(3), 133-155. doi: https://doi.org/10.1016/j.elstat.2006.07.012

Jaworek, A., Marchewicz, A., Sobczyk, A. T., Krupa, A., \& Czech, T. (2018). Two-stage electrostatic precipitators for the reduction of PM2.5 particle emission. Progress in Energy and Combustion Science, 67, 206-233. doi: https://doi.org/10.1016/j.pecs.2018.03.003

Kim, H., Kim, J., Yang, H., Suh, J., Kim, T., Han, B., . . Choi, M. (2006). Parallel patterning of nanoparticles via electrodynamic focusing of charged aerosols. Nature nanotechnology, 1(2), 117.

Koullapis, P. G., Kassinos, S. C., Bivolarova, M. P., \& Melikov, A. K. (2016). Particle deposition in a realistic geometry of the human conducting airways: Effects of inlet velocity profile, inhalation flowrate and electrostatic charge. Journal of Biomechanics, 49(11), 2201-2212. doi: https://doi.org/10.1016/j.jbiomech.2015.11.029 
Li, A., \& Ahmadi, G. (1992a). Dispersion and deposition of spherical particles from point sources in a turbulent channel flow. Aerosol Science and Technology, 16, 209-226. doi: $10.1080 / 02786829208959550$

Li, A., \& Ahmadi, G. (1992b). Dispersion and Deposition of Spherical Particles from Point Sources in a Turbulent Channel Flow. Aerosol Science Technology, 16, 209-226. doi: $10.1080 / 02786829208959550$

Li, A., \& Ahmadi, G. (1993a). Computer simulation of deposition of aerosols in a turbulent channel flow with rough wall. Aerosol Science Technology, 18, 11-24. doi: $10.1080 / 02786829308959581$

Li, A., \& Ahmadi, G. (1993b). Deposition of aerosols on surfaces in a turbulent channel flow. [doi: 10.1016/0020-7225(93)90017-O]. International Journal of Engineering Science, $31(3), 435-451$.

Longest, P. W., Kleinstreuer, C., \& Buchanan, J. R. (2004). Efficient computation of microparticle dynamics including wall effects. Computers \& Fluids, 33(4), 577-601.

Longest, P. W., \& Xi, J. (2007). Effectiveness of Direct Lagrangian Tracking Models for Simulating Nanoparticle Deposition in the Upper Airways. Aerosol Science and Technology, 41(4), 380-397. doi: 10.1080/02786820701203223

Majid, H., Winker-Heil, R., Madl, P., Hofmann, W., \& Alam, K. (2016). Effect of Oral Pathway on Charged Particles Deposition in Human Bronchial Airways. Journal of aerosol medicine and pulmonary drug delivery, 29(1), 24-29.

Malekian, D., Sajadi, B., Ahmadi, G., \& Pirhadi, M. (2018). A numerical study of electric force effects on detachment and deposition of particles due to a falling disk. Journal of Aerosol Science. 
Mayya, Y. S., Sapra, B. K., Khan, A., \& Sunny, F. (2004). Aerosol removal by unipolar ionization in indoor environments. Journal of Aerosol Science, 35(8), 923-941. doi: https://doi.org/10.1016/j.jaerosci.2004.03.001

Melandri, C., Tarroni, G., Prodi, V., De Zaiacomo, T., Formignani, M., \& Lombardi, C. C. (1983). Deposition of charged particles in the human airways. Journal of Aerosol Science, 14(5), 657-669. doi: http://dx.doi.org/10.1016/0021-8502(83)90070-8

Ounis, H., Ahmadi, G., \& McLaughlin, J. B. (1993). Brownian particles deposition in a directly simulated turbulent channel flow. Physics of Fluids A, 5, 1427-1432. doi: $10.1063 / 1.858578$

Ruzer, L. S., \& Harley, N. H. (2004). Aerosols Handbook: Measurement, Dosimetry, and Health Effects: CRC Press.

Schnelle Jr, K. B., Dunn, R. F., \& Ternes, M. E. (2015). Air pollution control technology handbook: CRC press.

Shy, C. M., Goldsmith, J. R., Hackney, J. D., Lebowitz, M. D., \& Menzel, D. B. (1978). Health effects of air pollution: American Thoracic Society, New York. Scientific Assembly on Environmental and Occupational Health.

Takasaki, M., Kurita, H., Kubota, T., Takashima, K., Hayashi, M., \& Mizuno, A. (2015). Electrostatic precipitation of diesel PM at reduced gas temperature. Paper presented at the Industry Applications Society Annual Meeting, 2015 IEEE.

Talebizadeh, P., Babaie, M., Brown, R., Rahimzadeh, H., Ristovski, Z., \& Arai, M. (2014). The role of non-thermal plasma technique in $\mathrm{NO} x$ treatment: A review. Renewable and Sustainable Energy Reviews, 40, 886-901.

Talebizadeh, P., Babaie, M., Kenny, E., Rahimzadeh, H., Inthavong, K., Ahmadi, G., \& Brown, R. (2015). Influence of pipe length and flow rate on nano-particle deposition in 
laminar circular pipe flows. International Journal of Energy and Environment, 6(4), 357366.

Talebizadeh, P., Rahimzadeh, H., Ahmadi, G., Brown, R., \& Inthavong, K. (2016). Time history of diesel particle deposition in cylindrical dielectric barrier discharge reactors. Journal of Nanoparticle Research, 18(12), 378.

Tian, L., \& Ahmadi, G. (2007). Particle Deposition in Turbulent Duct Flows - Comparisons of Different Model Predictions. Journal of Aerosol Science, 38(4), 377-397. doi: 10.1016/j.jaerosci.2006.12.003

Tsai, D. H., Kim, S. H., Corrigan, T. D., Phaneuf, R. J., \& Zachariah, M. R. (2005). Electrostatic-directed deposition of nanoparticles on a field generating substrate. Nanotechnology, 16(9), 1856.

Tu, G., Song, Q., \& Yao, Q. (2017). Experimental and numerical study of particle deposition on perforated plates in a hybrid electrostatic filter precipitator. Powder Technology, 321, 143-153. doi: https://doi.org/10.1016/j.powtec.2017.08.021

Tu, G., Song, Q., \& Yao, Q. (2018). Mechanism study of electrostatic precipitation in a compact hybrid particulate collector. Powder Technology, 328, 84-94. doi: https://doi.org/10.1016/j.powtec.2018.01.016

Tu, J., Inthavong, K., \& Ahmadi, G. (2012). Computational Fluid and Particle Dynamics in the Human Respiratory System: Springer Netherlands.

Wang, J., Flagan, R. C., \& Seinfeld, J. H. (2002). Diffusional losses in particle sampling systems containing bends and elbows. Journal of Aerosol Science, 33(6), 843-857.

Yu, K.-P., Shih, H.-C., Chen, Y.-C., \& Yang, X.-E. (2017). Effect of turbulence intensity and particle characteristics on the deposition of submicron particles enhanced by the ionic air purifier. Building and Environment, 114, 166-177. doi: https://doi.org/10.1016/j.buildenv.2016.12.023 
Zahmatkesh, I. (2008). On the importance of thermophoresis and Brownian diffusion for the deposition of micro- and nanoparticles. International Communications in Heat and Mass Transfer, 35(3), 369-375. doi: https://doi.org/10.1016/j.icheatmasstransfer.2007.08.004

Zheng, M., Reader, G. T., \& Hawley, J. G. (2004). Diesel engine exhaust gas recirculationa review on advanced and novel concepts. Energy Conversion and Management, 45(6), 883-900. doi: https://doi.org/10.1016/S0196-8904(03)00194-8

Zhou, Y., Zhong, S., \& Li, L. (2017). Characteristics and mechanisms of particle adhesion patterns in an aerodynamic cyclone. Aerosol Science and Technology, 51(11), 1313-1323. 